\title{
Zenithal bistability in a nematic liquid-crystal device with a monostable surface condition
}

\author{
L. A. Parry-Jones, ${ }^{\text {a) }}$ E. G. Edwards, S. J. Elston, and C. V. Brown \\ Department of Engineering Science, University of Oxford, Parks Road, Oxford, OX1 3PJ, \\ United Kingdom
}

(Received 13 August 2002; accepted 7 January 2003)

\begin{abstract}
The ground-state director configurations in a grating-aligned, zenithally bistable nematic device are calculated in two dimensions using a $Q$ tensor approach. The director profiles generated are well described by a one-dimensional variation of the director across the width of the device, with the distorted region near the grating replaced by an effective surface anchoring energy. This work shows that device bistability can in fact be achieved by using a monostable surface term in the one-dimensional model. This implies that is should be possible to construct a device showing zenithal bistability without the need for a micropatterned surface. (C) 2003 American Institute of Physics. [DOI: 10.1063/1.1557317]
\end{abstract}

In recent years, interest in bistable display technologies has grown dramatically in response to the increasing demand for portable devices with low power consumption. As such, zenithally bistable nematic devices (ZBNDs) are a highly promising display technology of the future. With a suitable surface relief structure on one of the inside surfaces of the cell, two stable director configurations [see Fig. 1(i)] can be supported, with the result that the device is bistable. ${ }^{1-5}$ This device, therefore, combines the advantage of a ferroelectric liquid-crystal display (namely lower power consumption when the display is infrequently updated), with the shock stability of nematic displays. Optical modeling of such devices ${ }^{6}$ has shown that the effect of a grating structure on one of the device surfaces is to reduce the contrast of the display. However, contrast ratios in excess of 15:1 have been achieved in demonstrator displays ${ }^{7}$ by optimizing the optical properties of the grating structure.

The director configurations shown in Fig. 1(i) have been generated with a two-dimensional (2D) $Q$ tensor modeling approach, ${ }^{8,9}$ using a sinusoidal grating with a depth of 0.8 $\mu \mathrm{m}$ and a pitch of $1.0 \mu \mathrm{m}$, in a device of total thickness 3.0 $\mu \mathrm{m}$. For these dimensions, the two stable states shown in Fig. 1(i) are of comparable energy. Although this is not necessary in order to achieve bistability, it optimizes the longterm stability of a static image in a display. Figure 1(ii) shows plots of the director tilt angle $\theta$ as a function of distance $z$ across the device width, at various points $x$ along the pitch of the grating. $\theta$ is defined to be zero when the director is parallel to the cell surfaces, and $\pi / 2$ when it is perpendicular to the surfaces. It is clear that the director structure shows negligible variation along the $x$ direction for $z<1.5 \mu \mathrm{m}$, and could therefore be described with a one-dimensional (1D) model along z. As shown in Fig. 1(iii), in one state (the vertical state), $\theta=\pi / 2$ throughout the device, whereas in the other state [the hybrid-aligned-nematic (HAN) state], the director tilts continuously from $\theta=\pi / 2$ to $\theta<\pi / 2$ across the device.

Davidson and Mottram have presented such a 1D model of a ZBND device ${ }^{10}$ in which the influence of the grating structure [i.e., the region $z>1.5 \mu \mathrm{m}$ in Fig. 1(ii)] is represented by an "effective surface energy" term

$$
F_{S}=\frac{W_{0}}{2} \sin ^{2} 2 \theta_{0}
$$

where $\theta_{0}$ is the liquid-crystal orientation at $z=d_{\text {effective }}$. This is of the form of a modified Rapini-Papoular term, ${ }^{11}$ and has equal energy minima at $\theta_{0}=0$ and $\theta_{0}=\pi / 2$, as illustrated by the curve for $W_{1} / W_{0}=0$ in Fig. 2(a). In the current work, the surface term will be considered to act at $z=d_{\text {effective }}$ $=d$, while the liquid-crystal orientation at $z=0$ is fixed at $\theta=\pi / 2$.

Assuming a one elastic constant approximation $\left(K_{11}\right.$ $=K_{22}=K_{33}=K$ ), a minimization of the Franck-Oseen elastic energy ${ }^{12-14}$ leads to a linear solution for $\theta$ :

$$
\theta(z)=\left(\frac{\theta_{0}-\pi / 2}{d}\right) z+\frac{\pi}{2}
$$

$\theta_{0}$ is given by balancing the torques due to the surface and elastic terms at the $z=d$ boundary, leading to the condition

$$
\sin 4 \theta_{0}=\frac{K}{W_{0} d}\left(\frac{\pi}{2}-\theta_{0}\right) .
$$

This equation always has at least one solution: $\theta_{0}=\pi / 2$, corresponding to the vertical state. In addition, when $K / W_{0} d$ $<0.87$, there are two other solutions which correspond to the HAN state and an unstable solution at the peak of the energy between the vertical and HAN states. According to Eq. (3), the maximum value of $\theta_{0}$ that can be obtained in the HAN state is 0.45 radians. However, Fig. 1(b,ii) shows that the value of $\theta_{0}$ predicted by the 2D model [for the grating in Fig. $1(\mathrm{~b}, \mathrm{i})]$ is larger than 0.45 radians.

Further, we note that according to the 1D model, the total energy of the device per unit area (integrated across the cell thickness) is

$$
\int F d z=\frac{K}{2 d}\left(\frac{\pi}{2}-\theta_{0}\right)^{2}+\frac{W_{0}}{2} \sin ^{2} 2 \theta_{0} .
$$

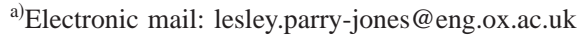




\section{(a) the "vertical" state}

(i)

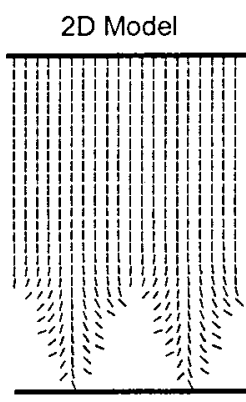

(ii)

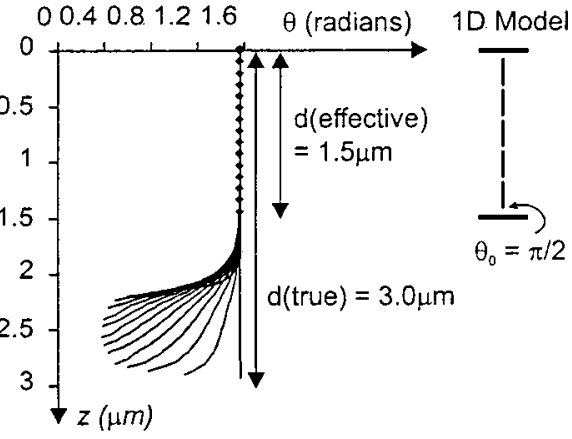

(b) the "HAN" state

(i)

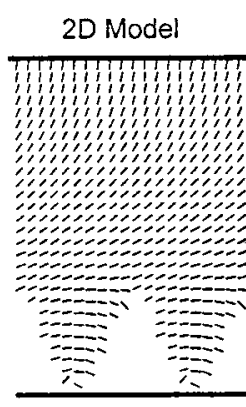

(ii)

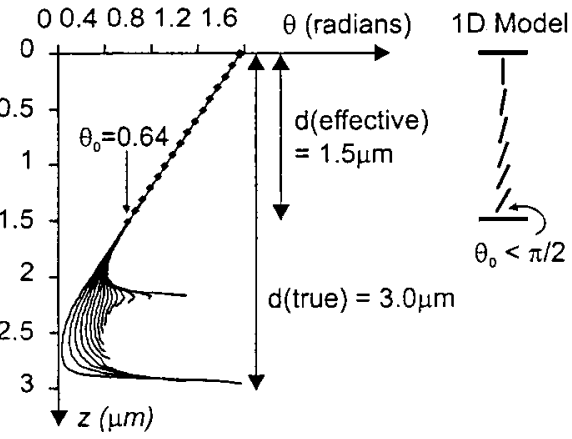

FIG. 1. Illustration of the two possible ground states that can exist in a ZBND, created using a surface relief structure, in this case, a grating of depth $0.8 \mu \mathrm{m}$ and pitch $1.0 \mu \mathrm{m}$ is used in a device of total thickness $3.0 \mu \mathrm{m}$. (a) In one state, the director just above the grating is essentially perpendicular to the glass plates, and hence the director structure can be simplified to one in which the director is vertical throughout the whole device: the "vertical" state. (b) In the other state, the director just above the device is almost planar. Thus, the simplified director structure is like that of a HAN state. Part (i) shows the director profile generated using a $Q$ tensor approach. Part (ii) shows slices taken through the 2D profiles: the "one-dimensional (1D) region" of the device can clearly be identified, as well as the effective value of $\theta_{0}$ in the HAN device. The results of a 1D model based on an effective surface term are shown by the discrete points. Part (iii) shows typical director profiles for the two states in the 1D model.

For the vertical state $\left(\theta_{0}=\pi / 2\right)$ the energy is zero, but for $\theta_{0}<\pi / 2$; that is, any other state including the stable HAN state, the energy is greater than zero. However, our 2D model shows that with the correct choice of grating and device dimensions, the two states can be comparable in energy. It is therefore clear that the influence of the grating structure is not represented fully by the surface term in Eq. (1).

In the 1D model, therefore, we propose to add to the existing surface energy expression a term that has an energy minimum at $\theta=0$ only, with a maximum at $\theta=\pi / 2$. The total surface energy term therefore becomes

$$
F_{s}=\frac{W_{0}}{2} \sin ^{2} 2 \theta_{0}+W_{1} \sin ^{2} \theta_{0}
$$

and is illustrated in Fig. 2(a) for $W_{1} / W_{0}>0$. Note that for $W_{1}<2 W_{0}$, the surface energy has two energy minima (at $\theta$ $=0$ and $\theta=\pi / 2$ ), and as such the surface is bistable, but for $W_{1}>2 W_{0}$, only the minimum at $\theta=0$ exists, that is, the surface is monostable. (a) Surface Energy (arb. units)

(b) Total Energy (arb. units)
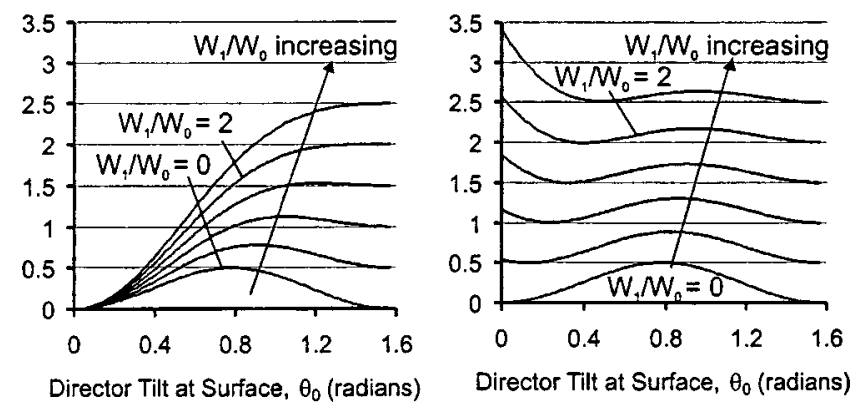

FIG. 2. Comparison of (a) surface and (b) total device energies, shown for a range of values of $W_{1} / W_{0}$. Note that it is possible for the whole device to be bistable, while the surface is monostable.

With the modified surface energy term, the equilibrium solution for $\theta$ is still as in Eq. (2), except that $\theta_{0}$ is now given by the solution to

$$
\sin 4 \theta_{0}+\frac{W_{1}}{W_{0}} \sin 2 \theta_{0}=\frac{K}{W_{0} d}\left(\frac{\pi}{2}-\theta_{0}\right),
$$

instead of Eq. (3). Some of the stable solutions now have much larger values of $\theta_{0}$, allowing correspondence with the results of the $2 \mathrm{D}$ model.

Using Eq. (5) in the 1D model, the total energy of the device per unit area is now given by

$$
\int F d z=\frac{K}{2 d}\left(\frac{\pi}{2}-\theta_{0}\right)^{2}+\frac{W_{0}}{2} \sin ^{2} 2 \theta_{0}+W_{1} \sin ^{2} \theta_{0} .
$$

For the vertical state $\left(\theta_{0}=\pi / 2\right)$ this reduces to $W_{1}$, instead of zero as before. It is therefore now possible for the HAN state $\left(\theta_{0}<\pi / 2\right)$ to be equal in energy to the vertical state. By equating the energies of the two states, and setting the torque on the surface director equal to zero [Eq. (6)], expressions for the dimensionless parameters $K / W_{0} d$ and $W_{1} / W_{0}$ can be obtained as a function of the surface tilt $\theta_{0}$. These expressions are plotted in Fig. 3, for the case of perfect device bistability. In order to achieve vertical and HAN states of equal energy, for any chosen value of $\theta_{0}$ the values of $W_{0}$ and $W_{1}$ (assuming fixed $K$ and $d$ ) are uniquely determined. The two equal energy states are stable minima that are separated by an energy barrier, as illustrated in Fig. 2(b).

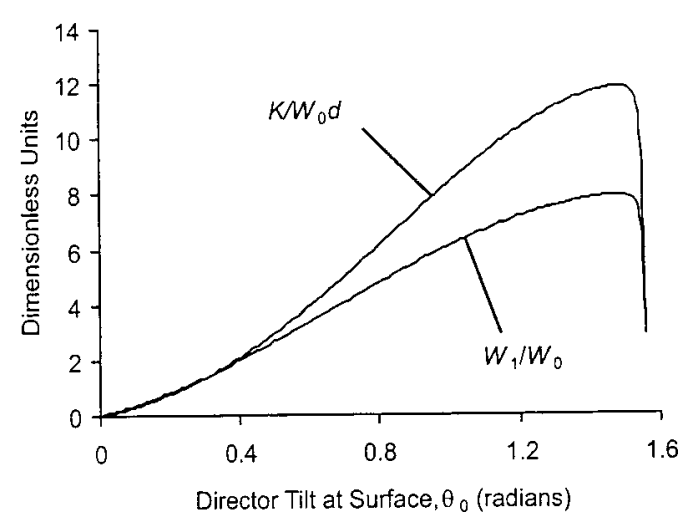

FIG. 3. If a modified surface energy term is used, and the vertical and HAN states are required to have the same energy, then the dimensionless parameters $K / W_{0} d$ and $W_{1} / W_{0}$ are uniquely determined for each value of $\theta_{0}$ (the director tilt angle at $z=d$ ). 
To illustrate this concept, the values of the effective surface energy coefficients $W_{0}$ and $W_{1}$ for the grating profile shown in Fig. 1(i) will be calculated. Figure 1(ii) shows that the effective 1D device thickness $d$ is $1.5 \mu \mathrm{m}$ (half the true device thickness of $3.0 \mu \mathrm{m}$ ), and $\theta_{0}=0.64$ radians. From Fig. 3, this gives

$$
\frac{K}{W_{0} d}=3.937
$$

and

$$
\frac{W_{1}}{W_{0}}=3.361
$$

at

$$
\theta_{0}=0.64 \text { radians. }
$$

Note that the value of $\theta_{0}$ obtained for a particular device depends on parameters other than the grating structure, that is, the total device thickness and the elastic constant. This means that the parameters $K / W_{0} d$ and $W_{1} / W_{0}$ are not independent of $d$ and $K$. Since $d=1.5 \mu \mathrm{m}$, and the director profile in Fig. 1(i) is generated using $K=1.55 \times 10^{-11} \mathrm{~N}$, then

$$
W_{0}=2.62 \times 10^{-6} \mathrm{~J} / \mathrm{m}^{2}, \quad W_{1}=8.82 \times 10^{-6} \mathrm{~J} / \mathrm{m}^{2} .
$$

The 1D director profiles generated using a numerical routine ${ }^{15}$ with these values of $W_{0}$ and $W_{1}$ are shown as the discrete points in Fig. 1(ii). It is anticipated that the 1D model can then be used to predict the switching characteristics of a ZBND with that grating, with the advantage of a lower computational burden than a $2 \mathrm{D}$ model.

For the case of the grating profile in Fig. 1(i), it is interesting to note that the value of $W_{1}$ required to represent the effect of the grating is such that $W_{1}>2 W_{0}$. In other words, even though the device is bistable, the surface energy itself is monostable. This can be understood as follows. In the vertical state, there is an energy cost at the surface because there is a surface energy maximum at $\theta=\pi / 2$. However, there is no energy cost in the bulk, because $\theta$ does not vary with $z$ and hence there is no stored elastic energy. In contrast, in the HAN state, the energy cost at the surface is low because $\theta_{0}$ is near the surface energy minimum at $\theta=0$. However, there is an energy cost in the bulk through the elastic energy stored in the change in $\theta$ from $\pi / 2$ at one surface to $\theta_{0}$ at the other. In our present analysis, the total surface and bulk energies have been balanced so that the total is the same in the two states.
It is therefore possible to have a bistable system in which the two states are equal in energy, even with a monostable surface. With a suitable choice of alignment method, therefore, it may be possible to achieve a zenithally bistable nematic device without the use of a surface relief structure on one surface. Possible candidates for suitable alignment layers include obliquely evaporated silicon oxide or a weakly rubbed polymer. Not only might such an alignment layer be simpler to fabricate than a grating structure, but the contrast of the device will be improved due to the elimination of diffractive effects caused by the periodic structure. It is also interesting to note that for the parameters used as an example, that is, a device of thickness $1.5 \mu \mathrm{m}$ with a surface tilt of 0.64 radians, if the liquid crystal were a typical nematic such as E7, the optical retardation of the device would be very close to the ideal quarter-wave condition, and hence give good contrast.

The authors would like to thank the EPSRC for financial support. Two of the authors (L.A.P.J.) and (S.J.E.) also gratefully acknowledge the financial support of the Leverhulme Trust, as does one of the authors (E.G.E.) to Hewlett-Packard Laboratories. We all thank A. Davidson and N. Mottram for useful discussions.

${ }^{1}$ G. D. Boyd, J. Cheng, and P. D. T. Ngo, Appl. Phys. Lett. 36, 556 (1980). ${ }^{2}$ J. Cheng, R. N. Thurston, G. D. Boyd, and R. B. Meyer, Appl. Phys. Lett. 40, 1007 (1982)

${ }^{3}$ E. Guyon, P. Pieranski, and M. Boix, Lett. Appl. Eng. Sci. 1, 19 (1984).

${ }^{4}$ G. P. Bryan-Brown, E. L. Wood, C. V. Brown, J. C. Jones, I. C. Sage, and P. Brett, Proceedings of the International Display Workshop. '97, 1997, p. 261.

${ }^{5}$ C. V. Brown, M. J. Towler, V. C. Hui, and G. P. Bryan-Brown, Liq. Cryst. 27, 233 (2000).

${ }^{6}$ E. E. Kriezis, C. J. P. Newton, T. P. Spiller, and S. J. Elston, Appl. Opt. 41, 5346 (2002)

${ }^{7}$ J. C. Jones, J. R. Hughes, A. Graham, P. Brett, G. P. Bryan-Brown, and E. L. Wood, Proceedings of the Seventh International Displays Workshop, Kobe, Japan, 2000, PLC2-2, p. 301.

${ }^{8}$ H. Mori, E. C. Gartland, J. R. Kelly, and P. J. Bos, Jpn. J. Appl. Phys. 38, 135 (1999).

${ }^{9}$ E. G. Edwards, S. C. Kitson, C. J. P. Newton, C. V. Brown, E. E. Kriezis, and S. J. Elston (unpublished).

${ }^{10}$ A. J. Davidson and N. J. Mottram, Phys. Rev. E 65, 051710 (2002).

${ }^{11}$ A. Rapini and M. Papoular, J. Phys. Colloq. 30, C4 (1969).

${ }^{12}$ C. W. Oseen, Trans. Faraday Soc. 29, 883 (1933).

${ }^{13}$ F. C. Franck, Discuss. Faraday Soc. 25, 19 (1958).

${ }^{14}$ J. L. Ericksen, Arch. Ration. Mech. Anal. 10, 189 (1962).

${ }^{15}$ C. V. Brown, L. A. Parry-Jones, S. J. Elston, and S. J. Wilkins (unpublished). 
Applied Physics Letters is copyrighted by the American Institute of Physics (AIP). Redistribution of journal material is subject to the AIP online journal license and/or AIP copyright. For more information, see http:/ojps.aip.org/aplo/aplcr.jsp

Copyright of Applied Physics Letters is the property of American Institute of Physics and its content may not be copied or emailed to multiple sites or posted to a listserv without the copyright holder's express written permission. However, users may print, download, or email articles for individual use. 